recovered in five weeks. This and the two subsequent attacks were all treated with an intravenous injection of $100,000 \mathrm{u}$. antitoxin, after precautions against reactions. On 19 November he had a severe recurrence of tetanus with all the classical symptoms and signs but more violent reflex spasms, which had to be controlled with inhalation of chloroform. It was three weeks before be could open his mouth, but he made a complete recovery.

He then remained well until October 1941, when he again developed trismus and signs of generalized chronic tetanus, without reflex spasms, from which he recovered in a few weeks. The rest of the story is best told in Mr. Wilson's own words: "At the time of his third attack the patient was not sure which thumb he had pricked, and I could find nothing on clinical or radiological examination. However, on consideration be felt it was his right, and I explored this. I removed a large wedge from the pulp of the right thumb, and to my great delight on opening this I found a large rose thorn. The patient never had any further tetanus and died aged 79."

It seems likely that these attacks of recurrent tetanus were due to a nidus of infection around the thorn embedded in the thumb.I am, etc.

Cambridge.

Leslie Cole.

\section{Primigravida or Primipara?}

SiR,-In reply to the letter from Dr. W. J. Elwood (20 January, p. 183) concerning methods of simply enumerating a pregnant woman's obstetric history, I personally no longer refer to the parity of the patient, because of the confusion which it can cause. I prefer to use the method which I think is in fairly widespread use of describing the gravid state, and breaking it down into confinements, including stillbirths, and miscarriages or abortions.

Thus a woman pregnant for the first time is described as (Gravid) G $1+0$. A woman pregnant for the second time, having previously had one confinement, would be described as G 2+0, whereas had she had one previous miscarriage and no previous confinement she would be described as G 1 +1 . A woman pregnant for the sixth time having had two miscarriages would be described as G $4+2$. It is easy to see at a glance what number the present pregnancy is simply by adding the confinements and miscarriages together, which, in this notation, are adjacent to one another.-I am, etc.,

$$
\begin{aligned}
& \text { Ilfracombe. } \\
& \text { North Devon. }
\end{aligned}
$$

A. J. BraY.

\section{Real Tennis Elbow}

SIR,-Strain of the common extensor origin at the lateral epicondyle due to lawn tennis is an uncommon cause of tennis elbow, as compared to direct or indirect trauma of an unsporting nature. "Table tennis elbow" and "badminton elbow" are also occasionally seen, but as far as I know the condition has never been described in connexion with real tennis, as in the following case.

A 34-year-old professional real tennis player bad recurrent pain in the region of the right elbow, which was severe enough to prohibit him playing in matches. On examination, he had the classical signs of lateral epicondylitis-that is, tenderness over the epicondyle, a positive Mills's sign, and pain on resistant dorsiflexion of the wrist while the elbow was extended. The condition responded satisfactorily to conservative treatment consisting of a hydrocortisone injection into the common extensor origin at the tenoperiosteal junction.

Real tennis, the forerunner of lawn tennis, is played on an enclosed court with a hard ball. The racket is roughly pear-shaped, one side being slightly flattened, giving it a rather asymmetrical appearance. The grip is similar to that used in lawn tennis, and the standard stroke is carried out with a chopping motion, rather like the volley stroke used in lawn tennis. The patient informs me that this necessitates greater strength of wrist and forearm than other court games, and therefore might well predispose to tennis elbow.I am, etc.,

$$
\begin{aligned}
& \text { Princess Alexandra Hospital, } \\
& \text { Harlow, Essex. }
\end{aligned}
$$

\section{Mental Health Education}

SIR,-I was interested in your leading article (13 January, p. 69) on mental health education, as we have recently completed a survey among a random sample of the adult Edinburgh population into attitudes and opinions regarding mental illness and the mentally ill.

The detailed results of the study are now in the press, but some features of the findings may be worthy of summary here.

The Edinburgh public proved to be generally familiar with the topic, either at first hand through personal experience or as a result of information from various sources, and they were prepared to discuss it frankly.

While it was true that many traces of old stereotypes of mental illness still persist, with connotations of possibly violent and unpredictable behaviour on the part of the mentally ill, there was evidence of relatively greater sympathy and tolerance among the younger and better-educated sections of the community. This may suggest that still further acceptance of psychiatric diagnosis and treatments are to be anticipated in the future, possibly posing problems for clinicians in the matter of case selection.

At the same time it appeared that certain personality factors such as neuroticism and a high regard for self-reliance are also involved in the extent of sympathy and tolerance which individuals feel towards the mentally ill and ex-mental patients.

It appeared that the picture of mental illness as projected by the mass media in Britain was tending to educate rather than to alarm the public. This is in contrast with the situation which has been reported from the United States. But there is still room for improvement in certain specific directions-for example, in the matter of information regarding current admission procedures, the relaxation of restraints within mental hospitals, and the expectation that periods of inpatient treatment will be short.

As research has shown that information levels are more susceptible to influence than attitudes, it would seem important to separate these two aspects of the problem and to concentrate attention upon the dissemination of correct information regarding what are nowadays the relatively optimistic prospects of treatment for mental illness.
The study in question was carried out from the Medical Research Council Unit for Research in the Epidemiology of Mental Illness at the Edinburgh University Department of Prychological Medicine.

-I am, etc.,

Department of Social Medicine, UNA MACLEAN.

University of Edinburgh.

\section{Errors in Blood Transfusion}

SIR,-Dr. D. A. Osborn once again proposes "eternal" vigilance as the price of safety in blood transfusion (2 December, p. 550). However, haemolytic transfusion reactions actually vary from a rate of about 1 in 1,000 in average hospitals to 1 in 10,000 in the most vigilant of hospitals. ${ }^{1}$ This is a sobering thought, particularly to anaesthetists, because general anaesthesia masks the signs of incompatible blood transfusion which would otherwise lead to an early recognition of incompatibility.

During an extensive operation for cancer six months ago a patient belonging to Group $\mathrm{O}$ received 5 pints $(2.8$ l.) of incompatible Group A blood due to at least two human errors. After 3 pints $(1.71$ ) of blood partial bronchospasm was noted and, soon after, some hypotension and generalized oozing from the surgical wound; however, only after 5 pints of blood had been administered did we manage to obrain laboratory confirmation of haemolytic transfusion reaction. The serum potassium level was $10.4 \mathrm{mEq} / 1$. and fatal ventricular fibrillation supervened while the patient was still in the operating theatre.

I report this case because since its occurrence we are able to give $100 \%$ assurance that such a catastrophe cannot recur in this institute. This claim is valid because we simply perform a duplicate micro-haematocrit from fingertip " capillary" blood after the first $200 \mathrm{ml}$. of blood transfused, and subsequently after each pint of blood transfused. The knowledge of the haematocrit is useful under the circumstances, but, in addition, the presence of more than $10 \mathrm{mg} . \%$ free plasma haemoglobin can be ruled out if macroscopic inspection of the supernatant plasma reveals clear plasma. ${ }^{2}$ If the plasma is at all tinged red the blood transfusion is stopped at once and chemical and spectrophotometric analysis for plasma haemoglobin is carried out.

It is surprising that this simple yet absolute safeguard against incompatible blood transfusion during general anaesthesia has not been described before.-I am, etc.,

$$
\begin{aligned}
& \text { Roswell Park Memorial J. W. MosteRT. } \\
& \text { IDstiute, } \\
& \text { Buffalo, New York } 14203 . \\
& \text { REFERENCEs } \\
& \text { ' Hardaway, R. M., in Surgical Bleeding, edised by } \\
& \text { A. W. Ulin and S. S. Gollub, 1966, P. 120. } \\
& \text { New York. } \\
& \text { 2 Mollison, P. L., Blood Transfusion in Chinical } \\
& \text { Medicine, 1967, 4th ed. Oxford. }
\end{aligned}
$$

\section{Salmonellosis in Terrapins}

SIR,-Referring to repeated warnings in the correspondence columns of the B.M.Y. (25 November, p. 486, 2 December, p. 551, and 16 December, p. 681), your readers might be interested to learn that, according to an investigation carried out by the U.S.A. Public Health Service, these "ornamental " terrapins are not caught in the wilds but are 